\title{
Omnidirectional photonic band gap in polymer photonic crystal slabs
}

\author{
Jan Hendrik Wülbern, ${ }^{a}$ Markus Schmidt, and Manfred Eich \\ Technische Universität Hamburg-Harburg, E-12, Eissendorfer Str. 38, D-21073 Hamburg, Germany \\ Uwe Hübner and Richard Boucher \\ Institute of Photonic Technology, A.-Einstein-Str. 9, D-07745 Jena, Germany \\ F. Marlow \\ Max-Planck-Institut für Kohlenforschung, Kaiser-Wilhelm-Platz 1, D-45470 Mülheim an der Ruhr, Germany \\ W. Volksen \\ IBM Almaden Research Center, 650 Harry Road, San Jose, California 95120 USA
}

(Received 21 September 2007; accepted 5 November 2007; published online 26 November 2007)

\begin{abstract}
In this letter, we report on the experimental observation of a complete in-plane photonic band gap for transverse-electric-like polarized modes in a polymer photonic crystal slab with a triangular array of holes. We performed transmission measurements on two-dimensional photonic crystal slabs in both principal directions of the triangular lattice. For a propagation distance of 40 lattice constants, the transmission was effectively suppressed regardless of propagation direction below $-15 \mathrm{~dB}$ over a bandwidth of $65 \mathrm{~nm}$. The experimental results are in excellent agreement with theoretical predictions obtained from band diagram calculations and finite integration time domain techniques. (c) 2007 American Institute of Physics. [DOI: 10.1063/1.2817331]
\end{abstract}

Photonic crystals $(\mathrm{PhCs})$ are structures in a dielectric material where the refractive index is modulated periodically in space. ${ }^{1,2}$ This results in a band structure for photons, similar to electrons in semiconductor lattices. By choosing an adequate geometry (lattice type and parameters), the band structure may be engineered in a way that a photonic band gap (PBG) is created and photons in a certain frequency range are Bragg reflected and, therefore, do not propagate in the crystal. By introducing defects into the periodic structure allowed states are created within the band gap, comparable to the effect of doping semiconductors.

$\mathrm{PhCs}$ may be distinguished by the number of dimensions in which the refractive index is altered periodically. Here, we are considering two-dimensional $\mathrm{PhCs}$ that are made from air holes etched into a slab waveguide that provides confinement of the light in the vertical direction by total internal reflection and allows interaction with the $\mathrm{PhC}$ in the plane of the slab. ${ }^{3}$ This type of $\mathrm{PhCs}$ is simpler to produce than threedimensional $\mathrm{PhCs}$ and have great potential as ultracompact integrated optical devices for narrow band filters formed by cavities, which are needed in wavelength division multiplexing applications as well as for components with tailored optical dispersion properties. It is attractive to realize such photonic integrated circuits in polymeric materials as they exhibit low losses at telecommunication wavelengths and simplify the coupling to silica fibers, due to similar refractive indices. Additionally, polymers may exhibit a strong nonlinear optical response, which can be used for electro-optic modulation. ${ }^{4}$ Due to the moderate refractive index of polymers $(n \approx 1.6)$, the index contrast to air is quite small, which is a situation not desirable for the design of structures with a complete PBG. However, it has recently been shown that polymer slabs immersed in air with a triangular array of holes can exhibit a complete PBG for transverse-electric (TE)-like polarization. ${ }^{5}$ The fact that in such air-bridge struc-

${ }^{a)}$ Electronic mail: jan.wuelbern@tu-harburg.de. tures, a major part of the waveguide does not rest on a solid substrate makes them intrinsically mechanically unstable and particularly imposes difficulties when a dielectric access channel is to be attached to the PBG defect waveguide. ${ }^{5}$

In this letter, we theoretically show and experimentally prove the existence of a complete PBG in a polymer structure that is not suspended in air, but uses an "airlike" substrate material with a refractive index close to 1 . Mesoporous silica is such a material due to its air filling fraction of $70 \%{ }^{6}$ Using this substrate, instead of air, offers two advantages. An additional wet etching step after structuring the waveguide core is not needed and the PhC slab has a good mechanical stability, since it resides on a solid substrate.

We used the guided mode expansion method to calculate the photonic band diagram of the $\mathrm{PhC}$ slab structure. ${ }^{7}$ Due to the vertical asymmetry of the structure, a strict distinction of TE and transverse-magnetic (TM)-polarized modes is not allowed. However, since the substrate has a very low refractive index $(n=1.15$ at $1300 \mathrm{~nm})$, which is close to the index of air the waveguide can be assumed to be "quasisymmetric." This allows the classification of the modes as being TE-or TMlike. In order to identify the TE- and TM-like polarized modes, we compared the field profiles of the threedimensional calculation to the results of two-dimensional (2D) calculations of the corresponding polarizations. The resulting band diagram is shown in Fig. 1. From this figure it can be seen, that a complete in-plane band gap exists for TE-like polarized modes between 0.51 and $0.53 \mathrm{c} / \mathrm{a}$. However, it can also be seen that only parts of this band gap are below the light line of the substrate (dashed line), which implies that modes with frequencies above the air band edge of the band gap can couple to radiation modes. Usually, it is desired to have PBG completely below the light line, since, otherwise, the lifetime, and hence the propagation distance of potential defect modes, could be significantly reduced by this coupling mechanism. As has been shown previously, this problem can be avoided by etching away the $\mathrm{PhC}$ underlying 


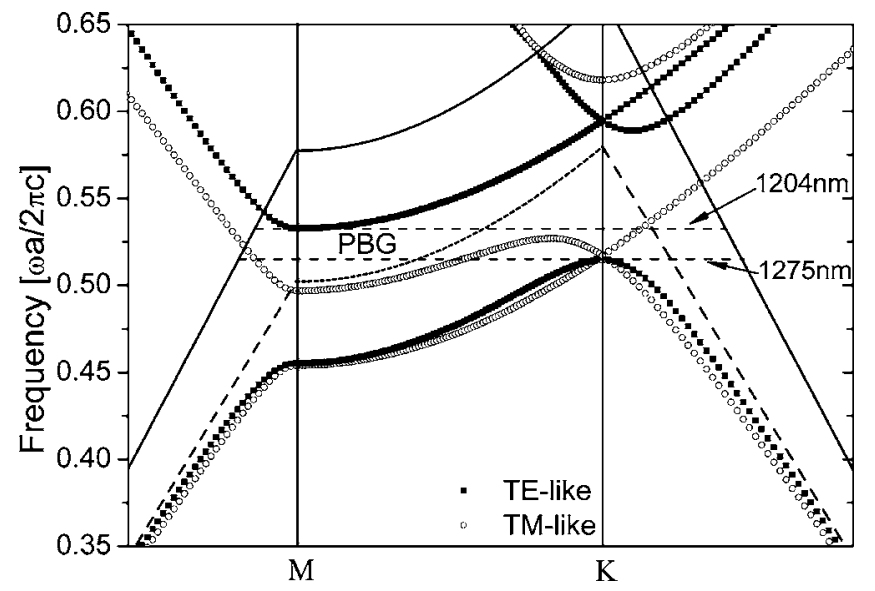

FIG. 1. Band diagram of a triangular photonic crystal of air holes in a polymer $(n=1.54)$ slab waveguide suspended on an airlike mesoporous silica material $(n=1.15)$. An omnidirectional PBG is visible for TE-like polarized modes (filled squares). The air and substrate light line are marked by solid and dashed lines, respectively. The arrows indicate the vacuum wavelengths at the edges of the complete band gap based on a lattice constant of $a=650 \mathrm{~nm}$.

substrate material, which would remove the substrate material and create an air-bridge structure. ${ }^{8}$

As we do not want significantly underetched waveguide structures for the reasons mentioned before, we will show below the losses incurred from vertical radiation to the continuum to be negligible in our experiment. Therefore, for this work, we omitted the additional fabrication step of etching away the substrate material underlying the $\mathrm{PhC}$. The guided mode expansion method, which was used for the photonic band calculation allows the extraction of the imaginary frequency part of the photonic bands that are above the light line. The imaginary frequency components describe radiation loss at a particular point in the band diagram, due to coupling to continuum modes above the light cone. In conjunction with the group velocity $\left(v_{g}\right)$, the radiation loss per unit length can be calculated. The propagation loss of a guided mode is given by $\alpha_{\text {loss }}=2 \operatorname{Im}(\omega) / \mathrm{v}_{\mathrm{g}}$. . Our calculations yielded that for our particular geometry these losses are below $1 \mathrm{~dB} / \mathrm{mm}$. Over a distance of a few tens (in our case 40) of lattice constants, the radiation losses are far below $1 \mathrm{~dB}$ and hence negligible as can be seen in Fig. 3. A removal of the substrate layer was therefore not necessary.

In the next step, we fabricated $\mathrm{PhC}$ slabs in order to experimentally confirm the existence of a complete in-plane PBG. Figure 2 shows a scanning electron microscopy picture of the investigated structures. The slab waveguides we used were made from low-index substrate mesoporous silica (thickness $1 \mu \mathrm{m}, n=1.15$ at $1300 \mathrm{~nm}$ excitation wavelength) and a polymer guiding layer of poly(methylmethacrylate) covalently functionalized with Disperse Red 1 P(MMA/DR1) (Ref. 10) (thickness $1.5 \mu \mathrm{m}, n=1.54$ at $1300 \mathrm{~nm}$ excitation wavelength) on silicon wafers (thickness of $0.54 \mathrm{~mm}$ ) by standard spin coating techniques. The photonic crystal structure is defined by electron beam lithography and reactive ion etching. ${ }^{11,12}$ We chose a triangular lattice with a lattice constant $a=650 \mathrm{~nm}$ and a hole radius of $280 \mathrm{~nm}$. The holes were etched into the core material (etching depth of $1.5 \mu \mathrm{m}$ ), with a slight penetration of the substrate layer. The bulk $\mathrm{PhCs}$ extended 40 lattice constants parallel to the direction of propagation and 8000 lattice constants perpendicular to it. In Downloaded 10 Aug 2010 to 134.28.141.212. Redistribution subject

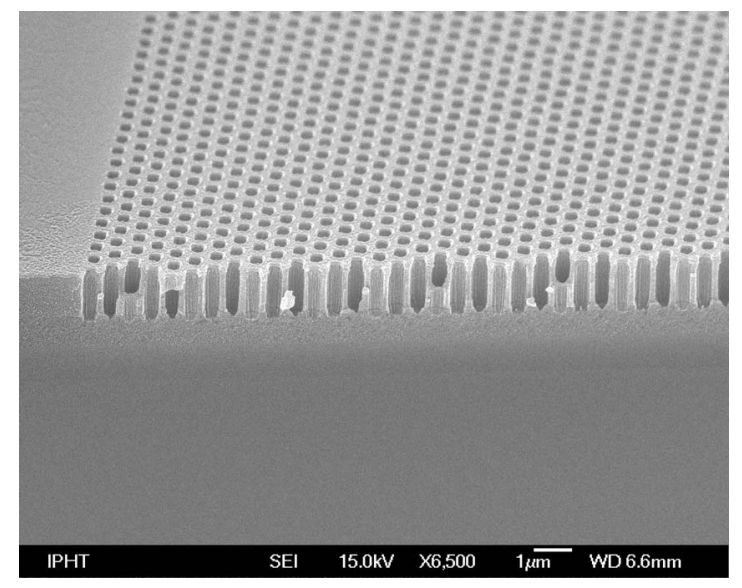

FIG. 2. Scanning electron micrograph displaying the cleaved edge of a $2 \mathrm{D}$ polymer triangular photonic crystal made from a $\mathrm{P}(\mathrm{MMA} / \mathrm{DR} 1)$ core, with mesoporous silica as the substrate material $(a=650 \mathrm{~nm}, r=280 \mathrm{~nm})$. Holes were etched into the core material, with a slight penetration of the substrate material.

order to allow measurements where the light could propagate in the $\Gamma M$ and $\Gamma K$ directions, two sets of structures were fabricated one for each propagation direction.

To measure the transmission spectra of the structures, a measurement setup was used in which the plane of polarization could be controlled. It consisted of a white light source (100 W halogen lamp), monochromator (1/4 m, excitation wavelength range of 600-2400 nm), Glan polarizer, and a Fresnel rhombus to select the desired polarization. The light was coupled in and out of the waveguide by means of prism couplers. The out coupled light was detected by a standard Ge diode and registered using a lock-in technique. All transmission spectra were measured with a resolution of $2 \mathrm{~nm}$ in a range from 1000 to $1600 \mathrm{~nm}$. The spectra of the patterned waveguide were divided by the spectra of the unpatterned waveguide in order to obtain the transmission characteristic of the $\mathrm{PhC}$. The results of these measurements are shown in Fig. 3, where the transmission spectra of the bulk PhC for each crystal orientation $(\Gamma M$ and $\Gamma K)$ are shown.

In the $\Gamma M$ direction, both the dielectric and air band edge (1400 and $1150 \mathrm{~nm}$, respectively) are clearly identifi-

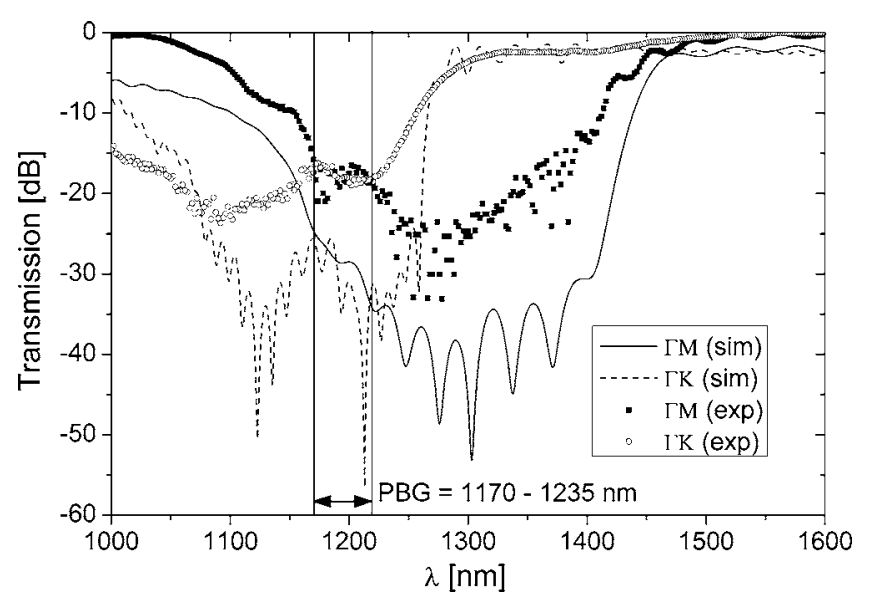

FIG. 3. Simulated (lines) and experimental (dots) transmission spectra of a bulk 40 lattice constant triangular photonic crystal in a polymer slab waveguide. The simulated and experimental results are in excellent agreement. A suppression of $15 \mathrm{~dB}$ represents the noise floor of the employed measurement apparatus. A band gap between 1170 and $1235 \mathrm{~nm}$ is clearly identifiable.

to AIP license or copyright; see http://apl.aip.org/apl/copyright.jsp 
able. The dielectric band edge in the $\Gamma K$ direction is also observable, whereas the air band edge is outside the measurement range. All these values compare very well to the results obtained from the guided mode expansion method calculations. Between 1170 and $1235 \mathrm{~nm}$, a region with a signal suppression of $-15 \mathrm{~dB}$ is clearly visible, which corresponds to the bandwidth of the omnidirectional PBG. The commercially available software package CST MICROWAVE STUDIO with its finite integration technique (FIT) code was used to calculate the expected transmission spectra. The result is plotted in Fig. 3 and is in very good agreement with the experimental results.

In summary, we fabricated bulk $\mathrm{PhCs}$ with a triangular lattice in polymer slab waveguides on low-index airlike substrates. We used e-beam lithography and a subsequent refractive ion etching step. Band diagram calculations predicted an omnidirectional PBG for TE-like polarized modes. This prediction was confirmed by transmission measurement in the $\Gamma M$ and $\Gamma K$ directions of the crystal. Furthermore, the experimental results show very good agreement with the results obtained from FIT calculations. For future applications, especially resonant structures and $\mathrm{PhC}$ waveguides realized in polymer materials, we will develop an etching method which allows the selective underetching of the PBG structure without the destabilization of the access waveguides. This is necessary in order to lift the light line completely above the
PBG and avoid losses from defect modes due to coupling to radiation modes.

This research was supported by the German Research Foundation DFG (EI 391/10-2). The authors acknowledge the support from CST, Darmstadt, Germany with their MICROWAVE STUDIO software.

${ }^{1}$ S. John, Phys. Rev. Lett. 58, 2486 (1987).

${ }^{2}$ E. Yablonovitch, Phys. Rev. Lett. 58, 2059 (1987).

${ }^{3}$ C. Liguda, G. Böttger, A. Kuligk, R. Blum, and M. Eich, Appl. Phys. Lett. 78, 2434 (2001).

${ }^{4}$ M. Schmidt, M. Eich, U. Hübner, and R. Boucher, Appl. Phys. Lett. 87, 121110 (2005).

${ }^{5}$ C.-S. Kee, S.-P. Han, K. B. Yoon, C.-G. Choi, H. K. Sung, S. S. Oh, H. Y. Park, S. Park, and H. Schift, Appl. Phys. Lett. 86, 051101 (2005).

${ }^{6}$ M. Schmidt, G. Boettger, M. Eich, W. Morgenroth, U. Huebner, R. Boucher, H. G. Meyer, D. Konjhodic, H. Breitinger, and F. Marlow, Appl. Phys. Lett. 85, 16 (2004).

${ }^{7}$ L. C. Andreani and D. Gerace, Phys. Rev. B 73, 235114 (2006).

${ }^{8}$ C.-G. Choi, Y.-T. Han, J. T. Kim, and H. Schift, Appl. Phys. Lett. 90, 221109 (2007)

${ }^{9}$ D. Gerace and L. C. Andreani, Opt. Express 13, 4939 (2005).

${ }^{10}$ We used P(MMA/DR1) because of its availability in large amounts and for the high film quality that we can achieve with this material. However, the procedures we have used can be easily applied to EO polymers with larger EO coefficients. We are currently working on this topic.

${ }^{11}$ J. H. Wülbern, M. Schmidt, U. Hübner, R. Boucher, W. Volksen, Y. Lu, R. Zentel, and M. Eich, Phys. Status Solidi A 204, 3739 (2007).

${ }^{12}$ U. Huebner, R. Boucher, W. Morgenroth, M. Schmidt, and M. Eich, Microelectron. Eng. 83, 1138 (2005). 